\title{
A Practical Approach to Subset Selection for Multi-objective Optimization via Simulation
}

\author{
CHRISTINE S. M. CURRIE, University of Southampton, UK \\ THOMAS MONKS, University of Exeter, UK
}

\begin{abstract}
We describe a practical two-stage algorithm, BootComp, for multi-objective optimization via simulation. Our algorithm finds a subset of good designs that a decision-maker can compare to identify the one that works best when considering all aspects of the system, including those that cannot be modeled. BootComp is designed to be straightforward to implement by a practitioner with basic statistical knowledge in a simulation package that does not support sequential ranking and selection. These requirements restrict us to a two-stage procedure that works with any distributions of the outputs and allows for the use of common random numbers. Comparisons with sequential ranking and selection methods suggest that it performs well, and we also demonstrate its use analyzing a real simulation aiming to determine the optimal ward configuration for a UK hospital.
\end{abstract}

CCS Concepts: • Computing methodologies $\rightarrow$ Simulation evaluation;

Additional Key Words and Phrases: Ranking and selection, simulation, subset selection, chance constraints

\section{ACM Reference format:}

Christine S. M. Currie and Thomas Monks. 2021. A Practical Approach to Subset Selection for Multi-objective Optimization via Simulation. ACM Trans. Model. Comput. Simul. 31, 4, Article 20 (August 2021), 15 pages.

https://doi.org/10.1145/3462187

\section{INTRODUCTION}

We consider a practical method for optimization via simulation in which a decision-maker wishes to choose the best of a large set of alternative designs. In practice, the decision over which design to choose is generally not based on just one outcome measure but instead is a multi-objective problem in which some aspects of the decision may not be incorporated into the simulation model. In these large, complex problems simulation is frequently used to reduce the set of possible designs to a smaller subset of good designs, which the decision-maker(s) can consider in more detail to identify which will work best in practice.

The method that we describe here is designed to be straightforward to implement such that an analyst with only a basic understanding of statistics and simulation modeling could use it. This

This report is independent research funded by the National Institute for Health Research Applied Research Collaboration Wessex. The views expressed in this publication are those of the authors and not necessarily those of the National Institute for Health Research or the Department of Health and Social Care.

Authors' addresses: C. S. M. Currie, University of Southampton, Highfield, Southampton, SO17 1BJ, UK; email: christine. currie@soton.ac.uk; T. Monks, University of Exeter, St Luke’s Campus, Heavitree Road, Exeter, EX1 2LU, UK; email: t.m.w.monks@exeter.ac.uk.

Permission to make digital or hard copies of part or all of this work for personal or classroom use is granted without fee provided that copies are not made or distributed for profit or commercial advantage and that copies bear this notice and the full citation on the first page. Copyrights for third-party components of this work must be honored. For all other uses, contact the owner/author(s).

(C) 2021 Copyright held by the owner/author(s).

1049-3301/2021/08-ART20 \$15.00

https://doi.org/10.1145/3462187 
places a first restriction on the method, that it should work for any distribution of the outputs, including correlated outputs. Making this restriction assumes that good practice such as the use of common random numbers can be followed but also that there is no fine print for the experimenter to read and comply with before running the method. Algorithms that place few restrictions on the output are typically described as general-purpose and bootstrapping, as used in BootComp, is often used to avoid constraints on the output distributions [Lee and Nelson 2014, 2015, 2016].

We also make the assumption, based on practical experience, that communication with the simulation model can be computationally expensive and difficult for a simulation practitioner to incorporate efficiently. This places a second restriction on the method, that the choice of designs to replicate over and the number of replications to make of each design can only be changed once during the experiment. Doing so rules out the fully sequential ranking and selection procedures that have dominated the literature in recent years and effectively enforces a two-stage process. We acknowledge that there are off-the-shelf simulation packages that include fully sequential ranking and selection procedures, but the majority do not and this procedure is designed to work well in those cases. The issue with building a ranking and selection algorithm that sits outside the software package is that there is likely to be a time delay for communication between the ranking and selection code and the simulation software, updating the model parameters, and (re)initializing the model. The "hidden cost" of communication time with the simulation model has previously been described in Kim and Nelson [2006a] but the difficulty of automating a simulation package to switch between the different designs being evaluated should not be underestimated, particularly where there are significant differences in the logic. In such situations, it is possible that the bootstrap ranking and selection procedures developed by Lee and Nelson may work well, as they suggest conducting $\Delta n$ observations at each step rather than just one [Lee and Nelson 2016]. An interesting practical problem would be to determine the optimal balance between the loss of efficiency that comes from using a high value of $\Delta n$ with the computational cost of communicating more frequently between the optimization software and the simulation software when $\Delta n$ is small.

A further benefit of the two-stage procedure is that it allows for efficient parallelization. Unlike the original sequential allocation algorithms, there is only a need to communicate results from different designs twice during the experiment. Where parallelization or cloud computing facilities are available this can result in a dramatic speedup in the computation times. Recent advances in sequential algorithms have addressed this issue (e.g., see Pei et al. [2018]) but BootComp allows for a straightforward treatment of parallelization.

Multi-objective problems have been considered in three ways previously in the ranking and selection literature: (i) using a Pareto front approach [Lee et al. 2010]; (ii) via chance constraints [Hong et al. 2015]; and (iii) optimizing a weighted average of the multiple objectives, e.g., see Swisher and Jacobsen [2002]. We use the second approach in which secondary objectives are reformulated as chance constraints, with the boundary values set based on discussions with system experts. While the Pareto approach has obvious merits, as it avoids the subjectivity inherent in the selection of threshold values for the chance-constraints, it can be difficult to explain to decisionmakers.

A Python implementation of the algorithm that we describe here is available for download [Monks and Currie 2020]. The algorithm relies on bootstrapping and, given the split into two stages, lends itself well to parallelization. An implementation using the cloud is available within the Github repository.

This article extends the work described in Monks and Currie [2018], in which we introduced the basic ideas of the method, with the most significant advance being a thorough comparison of its 
performance with OCBA-m on a standard set of problems frequently used to measure performance. We also extend the treatment of the hospital simulation model that was considered previously.

Summarizing the contributions described above, BootComp provides a unique approach to subset selection via simulation when there are multiple objectives. Its structure as a two-stage algorithm works well when communication between the simulation model and the optimization is computationally expensive or otherwise difficult. The procedure is also easy to implement: there are no restrictions placed on the distribution of the simulation output, and the fact that the algorithm has just two stages makes it both easy to parallelize and straightforward to use with an off-the-shelf simulation package.

We provide a formulation of the problem before the literature review and methods sections so the reader has a clear understanding of what we are aiming to achieve.

\subsection{Problem Formulation}

Assume there are in general $k \geq 2$ designs being compared and define $X_{i j}$ to be univariate, realvalued output data coming from replication $j$ for design $i$ that represents the main performance measure. We make no assumptions about the distribution of the $X_{i j}$ and allow for correlations between observations from different designs in the same replication, which may arise if CRNs are being used. We assume that observations from different replications are independent and identically distributed. Our main performance measure is assumed to be the mean of the output for a particular design after $n$ replications,

$$
\bar{X}_{i}=\sum_{j=1}^{n} X_{i j} / n,
$$

and we assume here that small values of $\bar{X}_{i}$ are desirable and that $\bar{X}_{i}$ is positive.

We also define a set of $l=1, \ldots, L$ secondary outputs, $Y_{i j l}$ coming from replication $j$ for design $i$. The $Y_{i j l}$ are real-valued and we assume that their mean values,

$$
\bar{Y}_{i l}=\sum_{j=1}^{n} Y_{i j l} / n,
$$

are the secondary objectives. The problem is written in such a way that large values of $\bar{Y}_{i l}$ are preferred. Our problem is one of subset selection with chance constraints on the secondary outputs. We wish to identify a shortlist or subset of designs $\mathrm{S}^{*}$ that contains designs where the expected value of the main performance measure is within a proportion $\beta$ of the expected value of the best feasible design, $\bar{X}^{*}$, with a probability $1-\alpha$, and fail the chance constraints with probability less than $\gamma$. Note that we interpret feasibility here as being feasible with respect to the constraints given above, ignoring any factors that cannot be modeled at this point. We also use a non-standard measure of proximity to the best in this work, preferring to use a proportion of the mean of the best design instead of the standard absolute difference. A design is said to satisfy the chance constraints if $\mathbb{P}\left\{\bar{Y}_{i} \geq 0\right\} \geq 1-\gamma$, where we use $\mathrm{Y}_{i}$ to represent the vector of sample means $\bar{Y}_{i l}, l=1, \ldots, L$. The main performance measure of the best feasible design $\bar{X}^{*}$ is defined as

$$
\bar{X}^{*}=\max _{i=1, \ldots, n} \bar{X}_{i} \text { such that } \mathbb{P}\left\{\bar{Y}_{i} \geq 0\right\} \geq 1-\gamma .
$$

Combining, our problem can be written as one of determining $\mathrm{S}^{*}$ such that

$$
\begin{aligned}
\mathbb{P}\left\{\bigcap_{i \in \mathrm{S}^{*}}\left(\bar{X}_{i} \leq \bar{X}^{*}(1+\beta)\right)\right\} & \geq 1-\alpha, \\
\mathbb{P}\left\{\overline{\mathrm{Y}}_{i} \geq 0\right\} & \geq 1-\gamma, i \in \mathrm{S}^{*} .
\end{aligned}
$$


The user and the decision maker set $m$ to be the maximum length of the shortlist. If the size of $\mathrm{S}^{*},\left|\mathrm{~S}^{*}\right|>m$, then the top $m$ designs in a ranked list of mean outputs of the key measure will be included in the shortlist. We make no claims that these will be the top $m$ designs, but only that they all lie within $\beta$ of the best feasible design with probability $1-\alpha$ and that there is a high probability that the designs we include in the final set are feasible. From a statistical perspective, this is the least satisfactory part of the method, but it helps in situations where there are a large number of suitable designs. An alternative would be to reanalyze the results with smaller values for $\beta$ or $\alpha$.

\section{LITERATURE REVIEW}

Selection procedures aim to find the best design or designs from a finite set of possibilities. Observations of each design are subject to stochastic variability, and the best design is generally that with the maximum or minimum mean. Branke et al. [2007] provides a thorough review of the topic and splits the approaches into three categories: the indifference zone approach; expected value of information procedures (VIP), and optimal computing budget allocation (OCBA). While indifference zone procedures such as Kim and Nelson [2006b] suggest sequential sampling plans that guarantee the probability of correct selection (PCS), OCBA procedures first introduced by Chen [1996] aim to maximize PCS with a fixed computation budget; and VIP methods choose which design to sample from based on the expected gain in the value of information [Chick and Inoue 2001b].

Parallelization and advances in cloud computing mean that there is a great deal of scope for speeding up these sequential selection procedures. For example, preliminary work in Pei et al. [2018] describes a framework Parallel Adaptive Survivor Selection (PASS), designed to exploit parallelization when solving ranking and selection problems with large numbers of designs. Instead of using PCS as an objective, PASS aims to minimize the expected false elimination rate, a statistic that scales better as the number of designs being tested increases. Methods that are able to easily transfer to parallel computing seem likely to dominate in the future.

The remainder of the literature review focuses on selection procedures designed for similar optimization problems to the one we consider here.

\subsection{Subset Selection}

Much of the literature on optimal subset selection aims to identify the best $m$ designs out of a total of $k$ possibilities, where the quality of a design is measured by its average output over all of the simulation replications. This raises an interesting question for users of the method as to what an appropriate value for $m$ might be and more flexible measures might be more appropriate, e.g., all designs within some percentage of the best. The difficulty with this latter approach is identifying the borderline for the subset when the mean output of the best design is unknown.

Early approaches to this problem used a two-stage approach. Sullivan and Wilson [1989] use a two-stage procedure to find a subset of designs such that the subset contains a design with mean that is less than $\delta$ from the best mean, with probability greater than or equal to a userdefined threshold. The two-stage procedure in Koenig and Law [1985] finds the subset of size $m$ that contains the best $m$ designs with probability at least equal to a threshold, providing that the difference between the objective of the $m+1$ th and $m$ th designs is no less than an indifference zone parameter. More recently, Wang et al. [2011] develop a sequential algorithm that finds a subset of designs that are either desirable or acceptable but not within the elimination region. These categories, which draw on ideas from indifference zone methods, are taken from Andradóttir and Kim [2010].

In the numerical examples considered later, we compare our algorithm with OCBA-m [Chen et al. 2008], which aims to find all of the top $m$ out of $k$ designs. There is a subtle difference between this 
procedure and earlier subset selection methods such as Gupta [1965], which aim to find a subset of designs that contain the best design with a pre-specified probability. The OCBASS algorithm introduced in Gao and Chen [2016] solves the same problem as OCBA-m, allocating replications to the design that is most likely to be "incorrectly observed." While this principle is similar to the standard OCBA algorithm, the application is subtly different as the question is now whether a design is within the boundary of the optimal subset or outside of it, rather than whether a design is the best or not. Numerical results suggest that OCBASS is more efficient than OCBA-m, with improvements in efficiency of around $29.2 \%$ and $42.5 \%$. Chingcuanco and Osorio [2013] consider the same problem but have a different approach in which they use all of the possible subsets of size $m$ as the different designs in a ranking and selection problem.

Gao and Chen [2015] instead minimize the Expected Opportunity Cost (EOC), measured as the difference in expected performance between the selected designs and the best designs. The use of EOC as a measure of the quality of a ranking and selection procedure was first introduced by Chick and Inoue [2001b] and has a clear practical benefit.

\subsection{Multi-objective Optimization via Ranking and Selection}

Hunter et al. [2019] provide a very clear introduction to multi-objective simulation optimization (MOSO), which has a wider scope than simply multi-objective ranking and selection problems. Within their discussion of ranking and selection procedures, they assume that these aim to identify the globally Pareto optimal designs, considering both fixed budget (e.g., MOCBA [Lee et al. 2010]) and fixed precision procedures. Globally Pareto optimal designs are considered to be those in the efficient set, i.e., no other design corresponds to an objective vector that is at least as small on all objectives and strictly smaller on at least one objective.

An alternative method for dealing with multi-objective problems is to instead reduce the dimensionality of the objective by introducing a multi-attribute variable that is a weighted average of the different objectives. A particularly relevant paper describing this approach, Swisher and Jacobsen [2002], aims to optimize the medical personnel staffing and facility size for a family practice clinic. Similar to the case study we describe at the end of this article, the measures of effectiveness of a policy include several elements and the authors identify appropriate weights to find a sum that best balances the tradeoffs between them before using the NM method [Nelson and Matejcik 1995] to carry out the optimization. The use of a weighted sum of objectives is a common method for addressing multi-objective optimization problems but one that introduces two potential issues: First, that it becomes harder to distinguish the individual contributions of different objectives in the weighted sum; and second, that the choice of weights is always somewhat arbitrary.

As discussed in the Introduction, we use chance constraints to account for secondary objectives, an approach that works well for many practical problems and can be easily explained to a decisionmaker. As far as we are aware, there are no other examples of algorithms that apply a two-stage procedure to identifying the optimal subset of designs with chance constraints when the output can take any distributional form. The main focus of the remainder of our review is on other algorithms that use chance constraints, as these are likely to be most closely related to the BootComp algorithm we describe here.

Hong et al. [2015] identify two distinct problems within optimization with chance constraints: Expectation Constrained Selection of the Best (ECSB) and Chance Constrained Selection of the Best (CCSB). In ECSB, the expected value of one or more outputs of the simulation model must be below some fixed value, while in CCSB, the constraints imposed by the decision-maker must be satisfied with a given probability. Andradóttir and Kim [2010], like us, use the expected value of the secondary output measure in the constraint equation, making this an ECSB problem. 
Their procedure uses an indifference zone to identify the best design that satisfies a single constraint. While they assume that the main and secondary outputs are normal, the results presented seem relatively robust to non-normality. Their approach to the chance constraint relies on Bechhofer's indifference zone structure [Bechhofer 1954] in which three different regions are identified for the value of the chance constraint: desirable, acceptable, and unacceptable.

Hong et al. [2015] instead solve the CCSB problem, and imposing this structure on the constraints facilitates checking the feasibility of designs. Their approach also assumes normality for the simulation outputs and selects just one optimal value. It allows for common random numbers (CRNs) and finds a relatively conservative solution for the number of replications due to its assumption that all chance constraints must be satisfied subject to the Bechhofer indifference zone structure.

Chance constraints have also been included in algorithms that aim to optimally allocate a fixed simulation budget in ranking and selection problems. For example, Pasupathy et al. [2014] make an elegant use of bilevel optimization to determine an algorithm SCORE to solve the ECSB problem; and Hunter and Pasupathy [2013] put forward a sequential algorithm that maximizes the asymptotic rate of decay of the probability of incorrect selection, reliant on knowing the underlying distribution of the output data.

\subsection{Generalized Ranking and Selection}

Many of the methods described in the previous sections make some assumption about the simulation outputs, the most common being independence and normality. Several authors have considered how ranking and selection can be achieved for more general distributions. Lee and Nelson [2016] describe their methods as general-purpose ranking and selection, and they allow for different output distributions for different designs; and measuring statistics other than the mean, as well as accounting for dependent observations (as observed when using CRNs) and non-normality. To provide guarantees of correct selection, Lee and Nelson [2016] use bootstrapping to estimate the probability that the observed best design is the best. When this exceeds the threshold, sampling can stop. Their method does not consider system constraints, a necessary feature of the BootComp algorithm we develop here. Bekki et al. [2010] have a slightly different aim of developing a flexible ranking and selection procedure that allows comparisons to be made on any distributional property. Again, the authors use non-parametric bootstrapping as a key part of their approach. The algorithm that we develop here also makes use of non-parametric bootstrapping as, although computationally intensive, bootstrapping is a straightforward way of allowing for general distributions.

\subsection{Two-stage Ranking and Selection Methods}

Articles on two-stage optimization methods were prevalent during the 1990s and the early 2000s following the publication of Koenig and Law [1985], who developed a two-stage procedure for subset selection. Of particular relevance to this work are Nelson and Matejcik [1995] and Chick and Inoue [2001a], who describe two-stage procedures that account for CRNs but for selection of a single design, rather than a subset. Both procedures assume normality in the outputs with Chick and Inoue [2001a] having a slightly less restrictive assumption about the covariance structure existing between the outputs of the different designs. The procedure described by Chick and Inoue [2001a] has more similarities to our work in that it works with a restricted budget and does not include designs in the second stage that it would be of no benefit to sample further. In contrast, the $N M$ procedure of Nelson and Matejcik [1995] aims to estimate the number of replications needed in stage 2 to achieve a threshold probability of correct selection. Nelson and Matejcik [1995] provide estimates of the difference between the best design and each of the other designs as a way 


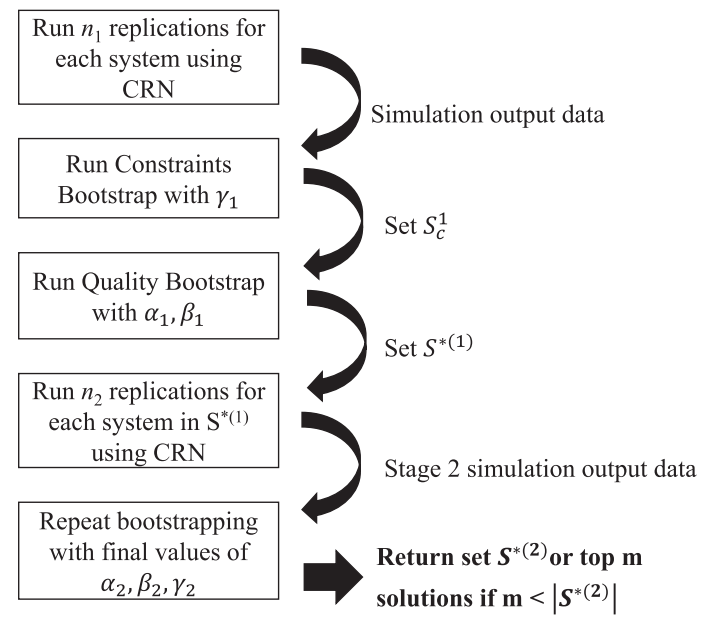

Fig. 1. An overview of the methodology.

of presenting a fuller set of results, allowing the decision-maker to consider other inferior designs that may have benefits not included in the simulation model. This goes some way towards addressing our assumption that decision-makers prefer to see a few possibilities rather than just one final result. Neither of these two-stage procedures considers multi-objective optimization, chance constraints, or non-normal output distributions.

\section{METHODOLOGY}

The method relies on two bootstrap routines, which are both run twice: first in stage one and again at the end of stage two. The first, Constraints Bootstrap, is used to determine the probability of a particular design violating the constraints, while the second, Quality Bootstrap, identifies the designs with means within a proportion $\beta$ of the mean of the best remaining designs. We begin by describing the whole procedure, which is also described in Figure 1, before giving more detailed descriptions of the two bootstrap routines.

(1) Run $n_{1}$ replications of the simulation model for all designs using CRNs if available to generate a set of primary outputs $X_{i j}$ and secondary outputs $Y_{i j l}$, where $j=1, \ldots, n_{1} ; i=1, \ldots, k$; $l=1, \ldots, L$. Run the Constraints Bootstrap described below in Section 3.1 to identify the set $\mathrm{S}_{c}^{1}$, which contains designs that have a probability of satisfying the chance constraints that is greater than $1-\gamma_{1}$. Inputs to the Constraints Bootstrap are $\mathrm{S}$, the complete set of designs, $\mathrm{Y}$, and $\gamma_{1}$.

(2) Run the Quality Bootstrap described below in Section 3.2 with inputs $\alpha_{1}, \beta_{1}, \mathrm{~S}_{c}^{1}$, and the $n_{1}$ by $k$ matrix of results for the main output measure $\mathrm{X}$. This outputs the set of designs $\mathbf{S}^{*(1)}$ that have means within a proportion $\beta_{1}$ of the best mean in set $S_{c}^{1}$ with probability greater than $1-\alpha_{1}$.

(3) Run $n_{2}$ replications of the simulation model for all designs in the set $\mathrm{S}^{*(1)}$ using CRNs if available.

(4) Follow steps 2 and 3 using data from both stages in $\mathrm{X}$ and $\mathrm{Y}$ and using parameters $\gamma_{2}, \alpha_{2}, \beta_{2}$. This results in a final shortlist of feasible and high-quality designs $\mathrm{S}^{*(2)}$.

(5) If the number of designs is greater than the desired number $m$, then pick the top $m$ in a non-decreasing list of means of the main output measure. Ties are decided using secondary output measures Y. 
We use different values for stage 1 and stage 2 parameters in the numerical results. These parameters are set by the user, and while we provide recommendations in Section 4.3, we acknowledge that more work is needed on determining optimal values.

\subsection{Constraints Bootstrap}

Our aim in the Constraints Bootstrap is to identify designs that are likely to violate the chance constraints. We assume that we have more than one chance constraint and use a fairly conservative measure of satisfying the chance constraints, as suggested in Hong et al. [2015] in which we insist that all of the constraints are satisfied simultaneously.

The routine takes as inputs $\mathrm{Y}$, the secondary outputs of the simulation model such that $Y_{i j l}$ is the value of the $l$ th secondary output, $l=1, \ldots, L$ in the $j$ th replication for the $i$ th design. Here, $i \in \mathrm{S}$ and $j=1, \ldots, n$. Note that $n=n_{1}$ in stage 1 and $n=n_{1}+n_{2}$ in stage 2 . Also input is $\gamma$, the threshold probability for failing the chance constraints.

As is standard with bootstrapping, we assume that $\mathrm{Y}$ can be viewed as a sample from a true multivariate distribution $G$ and use bootstrapping to sample from an approximation to this distribution, $\hat{G}$. The bootstrapping proceeds as follows:

(1) Generate $B$ bootstrap samples of size $n$ using a non-parametric bootstrap in which we sample with replacement from $Y$. This yields a set of bootstrap samples $Y^{\star(1)}, Y^{\star(2)}, \ldots, Y^{\star(B)}$ and for each of these we calculate $\bar{Y}_{i l}^{\star(b)}, l=1, \ldots, L$, where $\bar{Y}_{i l}^{\star(b)}=\frac{1}{n} \sum_{j=1}^{n} Y_{i j l}^{\star(b)}$.

(2) Include designs in the final feasible set $S_{c}$ if

$$
\frac{1}{B} \sum_{b=1}^{B} \prod_{l=1}^{L} I\left\{\overline{\mathrm{Y}}_{i l}^{\star(b)} \geq 0\right\} \geq 1-\gamma
$$

where $I(\cdot)$ is the indicator function, which takes a value of 1 if the condition inside the parentheses is true and 0 otherwise.

(3) Return $\mathrm{S}_{c}$.

\subsection{Quality Bootstrap}

The quality bootstrap aims to identify a set of designs $S^{*}$ that all have means within a distance $\beta$ of the mean of the best sampled design in $S^{*}$ with a probability $1-\alpha$. It takes as inputs $\alpha, \beta$, the set of feasible designs $\mathrm{S}_{c}$, and $\mathrm{X}$, a set of outputs for the main output measure $X_{i j}, i \in \mathrm{S}_{c}, j=1, \ldots, n$, where $n$ is the number of replications being considered in the bootstrap $\left(n_{1}\right.$ in stage 1 and $n_{1}+n_{2}$ in stage 2).

(1) Define a new random variable $\mathrm{D}$, with elements $D_{i j}, i \in \mathrm{S}_{c}, j=1, \ldots, n$, such that

$$
D_{i j}=X_{j}^{*}-X_{i j}
$$

where $X_{j}^{*}=X_{i^{*} j}, i^{*}=\arg \max \bar{X}_{i}$ is the value of the main output measure for the best, feasible design in stage 1 , in replication $j$ of the simulation. We can treat the set of differences $D_{i j}$ as a sample from the true multivariate distribution for the differences from the best, $F$.

(2) Generate $B$ bootstrap samples of size $n$ from $\hat{F}$, our approximation to $F$. We do this using a non-parametric bootstrap in which we sample with replacement from the set of $D_{i j}$. This gives us a new set of samples $\mathbf{D}^{\star(1)}, \mathbf{D}^{\star(2)}, \ldots, \mathbf{D}^{\star(B)}$, where each $\mathbf{D}^{\star(b)}$ is of the same dimensions as the original set of differences, $\mathrm{D}$. 
(3) Identify $S^{*}$ such that it is the biggest set for which

$$
\frac{1}{B} \sum_{b=1}^{B} \prod_{i \in S^{*}} I\left\{\frac{1}{n} \sum_{j=1}^{n} D_{i j}^{\star(b)} \geq-\beta \bar{X}^{*}\right\} \geq 1-\alpha .
$$

This final step is carried out by ordering the designs in non-increasing order of the main output measure and working down the list until Equation (3) is true for the set of designs at the top of the list but would not be true if the next design was included in the set.

(4) Return $S^{*}$.

\subsection{Python Implementation}

An implementation of the optimization procedure, BootComp [Monks and Currie 2020], was developed in Python 3.7.2 with dependencies NumPy 1.16.2, Pandas 0.24.1, SciPy 1.2.1, and Numba 0.42.0. Matplotlib 3.0.2 and Seaborn 0.9.0 were used for visualization in the analysis. All numerical examples, including a cloud executable example of using BootComp, are available online via GitHub and Binder (https://github.com/CLAHRCWessex/BootComp). For local installation, it is recommended that users install an Anaconda Distribution (https://www.anaconda.com/download/). Our code implementation includes an environment file that will install the appropriate dependencies. Users unfamiliar with version control or GitHub can simply download the repository and de-compress the files. Details of the applied example are found in the Jupyter notebook BootComp_Tutorial . ipynb.

\section{NUMERICAL EXAMPLES}

We begin this section by demonstrating that the algorithm performs well on a set of test problems considered in other articles introducing subset selection algorithms. It should be stressed that these test problems do not contain chance constraints, and we compare BootComp with a sequential algorithm, OCBA-m. As a result, we do not necessarily expect BootComp to be the top performer. Nonetheless, we feel that including the comparison provides a measure of its quality.

\subsection{Comparison to Single Objective OCBA-m}

We first compare our two-stage procedure to the Optimal Computing Budget Allocation (OCBA-m) procedure for returning the top $m$ designs [Gao and Chen 2016]. OCBA-m is a state-ofthe-art fully sequential single objective subset selection method. In our first test bed there are 10 competing designs whose outputs are normally distributed with mean $\mu_{1}=1, \mu_{2}=2 \ldots \mu_{10}=10$ and unit variance. In each experiment, the procedures are set to select the top three largest designs. The use of Common Random Numbers (CRNs) to reduce the variance between competing designs is standard within commercial off-the-shelf simulation software packages. We compare BootComp and OCBA-m over a range of different computational budgets (total number of replications), carrying out 10,000 experiments with and without the use of CRNs for each budget value. Respectively, this gives a sense of the best- and worst-case performance of BootComp.

The second test bed is the well-known inventory control problem introduced by Koenig and Law [1985] and also considered in Gao and Chen [2016]. In this example, we simulate 10,000 experiments of nine competing system designs in the Law inventory problem. CRNs have been employed, achieving $89 \%$ variance reduction between simulated designs. We consider two different optimization problems with this example: first, returning the three designs with the largest mean, and second, the two designs with the lowest mean.

Code and data to reproduce the numerical results are available in GitHub [Monks and Currie 2021]. 


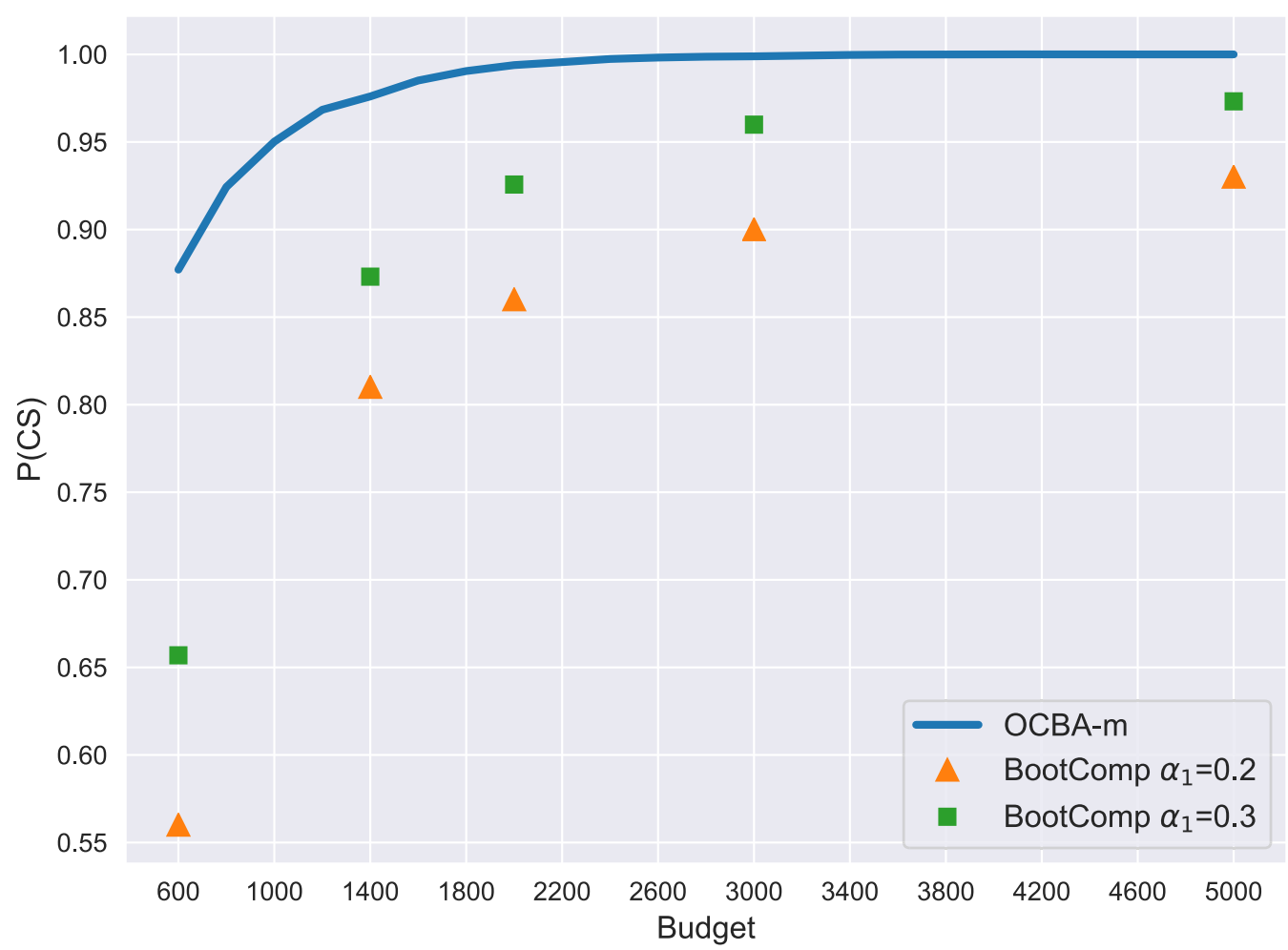

Fig. 2. Probability of correct selection of the top three designs in the 10 designs without CRNs case $(10,000$ experiments).

4.1.1 Ten Designs with CRNs. Both OCBA-m and BootComp were run with 20 initial replications for each design $\left(n_{1}=20\right)$. In all of the experiments, we use the following values for the BootComp parameters: $\alpha_{1}=0.2, \alpha_{2}=0.05, \beta_{1}=0.6, \beta_{2}=0.3$. We tested budgets between 300 and 2,000 and found that when competing designs use perfectly synchronized CRNs, BootComp correctly selected the top three designs in all 10,000 experiments for each budget. This result held with a more restrictive $\beta_{1}=0.4$. OCBA-m has high PCS in all experiments and selects the top three designs in all experiments when budgets exceed 800 replications.

4.1.2 Ten Designs without CRNs. Both OCBA-m and BootComp were run with 20 initial replications for each design $\left(n_{1}=20\right)$. In all of the experiments, we use the following values for the BootComp parameters: $\alpha_{1}=0.2, \alpha_{2}=0.05, \beta_{1}=0.6, \beta_{2}=0.3$. Again using 10,000 experiments, we tested budgets of between 600 and 5,000 replications. The comparison is illustrated in Figure 2 . The results illustrate the importance of CRNs in variance reduction and, as expected, BootComp loses its advantage over OCBA-m in examples where samples are independent. Without CRNs, BootComp was unable to match OCBA-m under any of the budget assumptions we included. This is not unexpected, as OCBA-m is a sequential algorithm and BootComp includes only two stages. We make two further observations. First, budgets above 1400 replications yielded a PCS with more utility for decision making. Second, selecting a higher value for $\alpha_{1}$ often yielded better results than holding $\alpha_{1}$ constant and increasing the replication budget.

4.1.3 Inventory Simulation Model. In the inventory example, full synchronization has not been achieved, but CRNs have still induced a high positive correlation across the nine designs. Both 
OCBA-m and BootComp select the top three designs (highest mean) in all 10,000 experiments for each budget. This result held when the problem was changed into a minimization to select the smallest two designs.

\subsection{Hospital Simulation Model}

As a final demonstration of the full method, including the chance constraints bootstrap, we apply BootComp to a problem of hospital ward design coming from a real project with the UK National Health Service (NHS). The project investigated the design of an NHS community hospital rehabilitation ward to minimize delays in the transfer of care of elderly in-patients from a large acute hospital. The new rehabilitation ward would be created from the merger of two geographically separate wards. The context and simulation model are described in detail in Penn et al. [2018], and the model built in Simul8 Professional 2018 is freely available online [Penn and Monks 2018].

NHS England mandates single-sex accommodation for patients. To accommodate this rule and provide some flexibility, beds within the wards could be put within single rooms or grouped in bays. Single rooms are in one sense the most efficient way to improve flow; however, single rooms are more expensive to safely staff. Bays of beds offer a good compromise to single rooms in terms of staffing costs and patient flow. An empty bay is assigned a gender corresponding to the gender of the first patient to be admitted. As patients are admitted and discharged from a ward, patients can be moved and the gender of the bay flipped to admit waiting patients. As there are no differences in process times for patients in a single room or within a bay, bays are less efficient for flow than single rooms. For example, a male patient requiring rehabilitation may not be able to be placed on a rehabilitation ward that has a free bed if the free bed is within a female designated bay. The simulation study, therefore, analyzed the mix of single rooms and size/number of bays that were required to keep waiting times for admission low and bed utilization acceptable.

The primary output of the model is the average waiting time for transfer to rehabilitation. Secondary outputs are utilization (occupancy) of beds and the number of patient transfers between gender-specific bays. Patient transfers are required when it is not possible to fit the current set of patients in the ward without breaking the constraint that a bay includes patients of the same gender. The client requested a set of good designs as opposed to a single optimal design.

\subsection{Experiments and Comparisons}

In stage one, the model was used to conduct 1,151 initial simulation experiments (stage 1 replications $n_{1}=5$; time unit = days; run length $=365$ days). The replications can be found in three .csv files in the Git repository (data\replications_wait_times.csv; data\replications_util.csv; data \replications_transfers.csv).

Table 1 lists the parameters used in the two-stage optimization. Clients preferred an average utilization of at least $80 \%$, and the upper threshold for the mean number of patient transfers between bays was set to 50 . The number of replications in stage 1 is based on the pragmatic recommendations from Law and Kelton [2000]; taking the upper bound of their recommended range of at least 3-5 replications. In the applied example, it took approximately 2.5 hours to generate the stage one replications for all 1,151 designs, using commercial software and a machine of a similar specification that would be found in use in industry (Simul8 Professional 2017; Dell Laptop; 16GB RAM; i7 processor). Chernick [2007] warns of the limitations of the bootstrap for sample sizes below 10. We found that results were consistent if stage one replications were extended to 10 per design (with the associated 5-hour model runtime) and consequently report the results with just 5 replications. The stage 1 and stage 2 values for $\gamma$ were chosen to allow for the fact that errors will be higher in stage 1 due to the smaller number of replications, while $\alpha$ was set to 0.05 to mimic accepted practice in ranking and selection procedures and $\beta$ to 0.05 based on the clients' preferences. We set $n_{2}$ 
Table 1. Parameters Used in the Optimization

\begin{tabular}{|l|c|c|}
\hline Parameter & Stage 1 & Stage 2 \\
\hline Utilization constraint & $\geq 80 \%$ & $\geq 80 \%$ \\
Patient transfers & $\leq 50$ & $\leq 50$ \\
Replications & 5 & 50 \\
$\gamma$ & 0.7 & 0.95 \\
$\alpha$ & 0.05 & 0.05 \\
$\beta$ & 0.3 & 0.05 \\
\hline
\end{tabular}
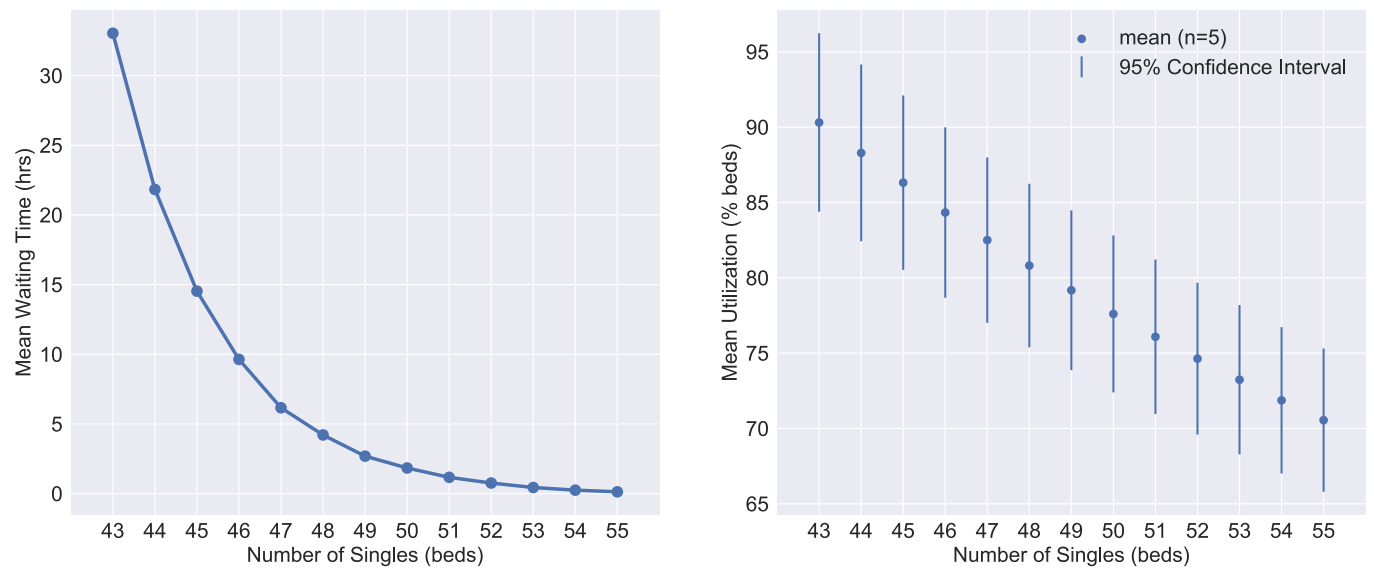

Fig. 3. Illustration of stage 1 utilization chance constraint.

based on the total computation time available and the number of designs being passed through to stage $2\left|S^{*(1)}\right|$.

\subsection{Chance Constraints}

Figure 3 illustrates an initial informal analysis of the conflict between the mean waiting time (Figure 3(a)), which is the main output measure, and the first chance constraint, which requires utilization to be greater than $80 \%$ (Figure $3(\mathrm{~b})$ ). For reasons of simplicity, the chart only includes results for designs that have exclusively single rooms and with the number of beds in the range 43-55.

\subsection{Optimization Procedure}

Figure 4 illustrates our optimization algorithm applied to the rehabilitation model. Stage 1 reduces the number of designs from 1,151 to 37. Most of this reduction in designs is delivered by the chance constraints - reducing the feasible set to 175 . A further 45 replications were conducted in stage 2 , resulting in a total of 7,285 individual runs of the model in stages 1 and 2. Stage 2 reduced the number of designs from 37 to 28 . Table 2 lists the top 10 final elite designs. These all have 48 beds, and suggest that bay sizes of 3 or 4 are optimal.

\section{CONCLUSION}

Our aim in this work was to develop a tool for multi-objective simulation optimization that is simple-to-use, fits within typical simulation practice, does not require the user to implement code to interact with the simulation models, and yet draws on recent research in ranking and selection. 


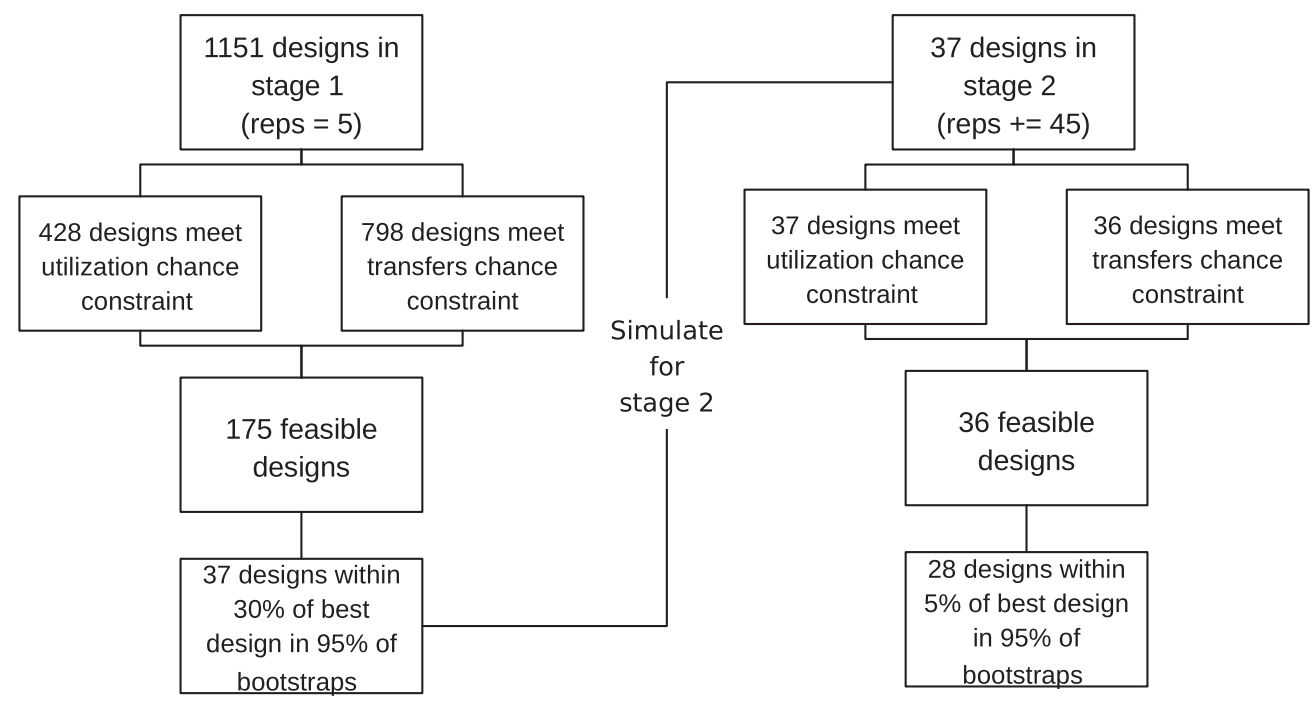

Fig. 4. Optimization procedure in applied example.

Table 2. Details of the Top 10 Scenarios

\begin{tabular}{|c|c|c|c|c|c|c|}
\hline Total Beds & Bay Size & $\begin{array}{c}\text { Number of } \\
\text { Bays }\end{array}$ & $\begin{array}{c}\text { Number of } \\
\text { Singles }\end{array}$ & $\begin{array}{c}\text { Waiting Time } \\
\text { (hours) }\end{array}$ & Utilization & Transfers \\
\hline 48 & 0 & 0 & 48 & 4.9 & 81.0 & 0.0 \\
48 & 3 & 3 & 39 & 4.9 & 81.0 & 12.7 \\
48 & 3 & 4 & 36 & 4.9 & 81.0 & 16.5 \\
48 & 3 & 5 & 33 & 4.9 & 81.0 & 20.9 \\
48 & 3 & 6 & 30 & 4.9 & 81.0 & 24.4 \\
48 & 3 & 7 & 27 & 4.9 & 81.0 & 29.9 \\
48 & 3 & 8 & 24 & 4.9 & 81.0 & 35.3 \\
48 & 3 & 9 & 21 & 4.9 & 81.0 & 40.2 \\
48 & 4 & 2 & 40 & 4.9 & 81.0 & 12.5 \\
48 & 4 & 3 & 36 & 4.9 & 81.0 & 17.3 \\
\hline
\end{tabular}

The result is a two-stage method, which reduces simulation effort by removing designs that are likely to be infeasible or poorly performing at the end of the first stage, and goes on to return a subset of designs that satisfy one or more chance constraints and are within a fixed tolerance of the best design.

The article includes a full comparison of the method on test problems within subset selection, and these results suggest that BootComp performs well in simulation studies where CRNs have been employed and have either fully synchronized or induced a positive correlation. Our experience is that many simulation practitioners use commercial simulation software that employs CRNs. We have also demonstrated our procedure's use on a complex simulation from health care, showing how it can be used in practice. The first stage appears to be very effective at significantly reducing the number of designs in the second-stage comparison.

We have made available an efficient Python implementation of BootComp. We hope that this will encourage further testing of the method and subsequent improvements as well as its use in practice. The framework exploits rudimentary parallelism and for large solution spaces (or 
for those where the user wishes to run a very high number of bootstraps) it can make use of multi-core CPUs to reduce runtime. Parallelization of the Constraints Bootstrap is straightforward, as it deals with marginal probabilities whereas parallelization of the Quality Bootstrap is more difficult. Parallelizing these bootstrap routines does not give substantial increases in efficiency compared with parallelizing the simulation.

One of the disadvantages of the method in its current form is that the user is required to decide on a number of different parameters for threshold probabilities, both for satisfying chance constraints and for being within a given tolerance of the best design. In the second stage, these parameters have some intrinsic meaning, but setting their values in the first stage is not straightforward and may require some experimentation. We suggest $\gamma_{1}>\gamma_{2}$. Parameters $\alpha_{1}$ and $\beta_{1}$ should generally be chosen to be greater than $\alpha_{2}$ and $\beta_{2}$ to ensure that competitive designs are not discarded in stage 1 . In the applied example considered here, we found that increasing the value of $\gamma_{1}$ (the threshold value for failing the chance constraints in stage 1) from 0.7 to 0.8 led to a different set of elite designs. More experimentation is needed to determine more clearly how the choice of these parameters affects the final design. One of the benefits of this method is that there is scope for experimentation with stage 1 parameters before running stage 2 replications; for example, if the initial choice of parameters suggests either very few or very many designs remain in the process.

Further work could consider how this two-stage approach could be adapted to identify the Pareto set of designs rather than treating the secondary objectives as chance constraints. There is some appeal in doing this, particularly for problems in which there is no objective that is clearly more important than the others.

\section{REFERENCES}

S. Andradóttir and S.-H. Kim. 2010. Fully sequential procedures for comparing constrained systems via simulation. Nav. Res. Logist. 57 (2010), 403-421.

R. E. Bechhofer. 1954. A single-sample multiple decision procedure for ranking means of normal populations with known variances. Ann. Math. Statist. 25 (1954), 16-39.

J. M. Bekki, B. L. Nelson, and J. W. Fowler. 2010. Bootstrapping-based fixed-width confidence intervals for ranking and selection. In Proceedings of the Winter Simulation Conference, B. Johansson et al. (Eds.). IEEE, Piscataway, NJ, 1024-1033.

J. Branke, S. E. Chick, and C. Schmidt. 2007. Selecting a selection procedure. Manag. Sci. 53 (2007), 1916-1932.

C.-H. Chen. 1996. A lower bound for the correct subset-selection probability and its application to discrete event simulations. IEEE Trans. Automat. Control 41 (1996), 1227-1231.

C.-H. Chen, D. He, M. Fu, and L. H. Lee. 2008. Efficient simulation budget allocation for selecting an optimal subset. INFORMS f. Comput. 20 (2008), 579-595.

M. R. Chernick. 2007. Bootstrap Methods: A Guide for Practitioners and Researchers. John Wiley \& Sons, Inc, NY.

S. E. Chick and K. Inoue. 2001a. New procedures to select the best simulated system using common random numbers. Manag. Sci. 47 (2001), 1133-1149.

S. E. Chick and K. Inoue. 2001b. New two-stage and sequential procedures for selecting the best simulated system. Oper. Res. 49 (2001), 732-743.

F. Chingcuanco and C. Osorio. 2013. A procedure to select the best subset among simulated systems using economic opportunity cost. In Proceedings of the Winter Simulation Conference, R. Pasupathy et al. (Eds.). IEEE, Piscataway, NJ, 452-462.

S. Gao and W. Chen. 2015. A note on the subset selection for simulation optimization. In Proceedings of the Winter Simulation Conference, L. Yilmaz et al. (Eds.). IEEE, Piscataway, NJ, 3768-3776.

S. Gao and W. Chen. 2016. A new budget allocation framework for selecting top simulated designs. IIE Trans. 48 (2016), $855-863$.

S. S. Gupta. 1965. On some multiple decision (selection and ranking) rules. Technometrics 7 (1965), 225-245.

L. J. Hong, J. Luo, and B. L. Nelson. 2015. Chance constrained selection of the best. INFORMS f. Comput. 27 (2015), 317-334.

S. R. Hunter, E. A. Applegate, V. Arora, B. Chong, K. Cooper, O. Rincón-Guevara, and C. Vivas-Valencia. 2019. An introduction to multiobjective simulation optimization. ACM Trans. Model. Comput. Simul. 29, 1 (2019). DOI: https: //doi.org/10.1145/3299872

S. R. Hunter and R. Pasupathy. 2013. Optimal sampling laws for stochastically constrained simulation optimization on finite sets. INFORMS f. Comput. 25 (2013), 527-542. 
S.-H. Kim and B. L. Nelson. 2006a. Selecting the best system. In Handbooks in Operations Research and Management Science: Simulation, Shane G. Henderson and Barry L. Nelson (Eds.). Vol. 13. Elsevier B.V., North Holland.

S.-H. Kim and B. L. Nelson. 2006b. Selecting the best system. In Elsevier Handbooks in Operations Research and Management Science: Simulation, S. G. Henderson and B. L. Nelson (Eds.). Elsevier, Waltham, MA.

L. W. Koenig and A. M. Law. 1985. A procedure for selecting a subset of size $\mathrm{m}$ containing the $\mathrm{l}$ best of $\mathrm{k}$ independent normal populations. Commun. Statist. - Simul. Computat. 14 (1985), 719-734.

A. M. Law and W. D. Kelton. 2000. Simulation Modeling \& Analysis (3rd ed.). McGraw-Hill, Inc, New York.

L. H. Lee, E. K. Pen Chew, S. Teng, and D. Goldsman. 2010. Finding the non-dominated Pareto set for multi-objective simulation models. IIE Trans. 42 (2010), 656-674.

S. Lee and B. L. Nelson. 2014. Bootstrap ranking and selection revisited. In Proceedings of the Winter Simulation Conference, A. Tolk et al. (Eds.). IEEE, Piscataway, NJ, 3857-3868.

S. Lee and B. L. Nelson. 2015. Computational improvements in bootstrap ranking and selection procedures via multiple comparison with the best. In Proceedings of the Winter Simulation Conference, L. Yilmaz et al. (Eds.). IEEE, Piscataway, NJ, 3758-3767.

S. Lee and B. L. Nelson. 2016. General-purpose ranking and selection for computer simulation. IIE Trans. 48 (2016), 555-564.

T. Monks and C. S. M. Currie. 2018. Practical considerations in selecting the best set of simulated systems. In Proceedings of the Winter Simulation Conference, M. Rabe, A. A. Juan, N. Mustafee, A. Skoogh, S. Jain, and B. Johansson (Eds.). IEEE, Piscataway, NJ, 2191-2200.

T. Monks and C. S. M. Currie. 2020. CLAHRCWessex/BootComp: v1.0.1. DOI : https://doi.org/10.5281/zenodo.3901489

T. Monks and C. S. M Currie. 2021. CLAHRCWessex/subset-selection-problem: v1.0.1. DOI : https://doi.org/10.5281/zenodo. 4552522

B. L. Nelson and F. J. Matejcik. 1995. Using common random numbers for indifference-zone selection and multiple comparisons in simulation. Manag. Sci. 41 (1995), 1935-1945.

R. Pasupathy, S. R. Hunter, N. A. Pujowidianto, L. H. Lee, and C.-H. Chen. 2014. Stochastically constrained ranking and selection via SCORE. ACM Trans. Model. Comput. Simul. 25 (2014), 1-26.

L. Pei, S. Hunter, and B. L. Nelson. 2018. A new framework for parallel ranking \& selection using an adaptive standard. In Proceedings of the Winter Simulation Conference, M. Rabe, A. A. Juan, N. Mustafee, A. Skoogh, S. Jain, and B. Johansson (Eds.). IEEE, Piscataway, NJ, 2201-2212.

M. L. Penn and T. Monks. 2018. Generic Ward Configuration Simulation Model. DOI : https://doi.org/10.5281/zenodo.1468288

M. L. Penn, T. Monks, A. Kazmierska, , and M. Alkoheji. 2018. Designing and redeveloping generic models in healthcare. In Proceedings of the OR Society Simulation Workshop, A. Anagnostou et al. (Eds.). OR Society, Stratford-Upon-Avon, UK.

D. W. Sullivan and J. R. Wilson. 1989. Restricted subset selection procedures for simulation. Oper. Res. 37(1) (1989), 52-71.

J. R. Swisher and S. H. Jacobsen. 2002. Evaluating the design of a family practice healthcare clinic using discrete-event simulation. Health Care Manag. Sci. 5 (2002), 75-88.

Y. Wang, L. Luangkesorn, and L. J. Shuman. 2011. Best-subset selection procedure. In Proceedings of the Winter Simulation Conference, S. Jain et al. (Eds.). IEEE, Piscataway, NJ, 4315-4323.

Received September 2020; revised June 2020; accepted March 2021 